Published in final edited form as:

Sex Transm Dis. 2014 February ; 41(2): 123-128. doi:10.1097/OLQ.0000000000000075.

\title{
Bacterial vaginosis and the risk of Trichomonas vaginalis acquisition among HIV-1 negative women
}

\author{
Jennifer E. Balkus ${ }^{1,2}$, Barbra A. Richardson ${ }^{1,2,3}$, Lorna K. Rabe ${ }^{4}$, Taha E. Taha ${ }^{5}$, Nyaradzo \\ Mgodi $^{6}$, Margaret Phiri Kasaro ${ }^{7}$, Gita Ramjee ${ }^{8}$, Irving F. Hoffman ${ }^{9}$, and Salim S. Abdool \\ Karim ${ }^{10,11}$ \\ ${ }^{1}$ Vaccine and Infectious Disease Division, Fred Hutchinson Cancer Research Center; Seattle, \\ WA, USA \\ ${ }^{2}$ Department of Global Health, University of Washington; Seattle, WA, USA \\ ${ }^{3}$ Department of Biostatistics, University of Washington; Seattle, WA, USA \\ ${ }^{4}$ Magee-Womens Research Institute; Pittsburgh, PA, USA \\ ${ }^{5}$ Department of Epidemiology, Johns Hopkins Bloomberg School of Public Health; Baltimore, MD, \\ USA
}

${ }^{6}$ Department of Obstetrics and Gynecology College of Health Science, University of Zimbabwe; Harare, Zimbabwe

${ }^{7}$ Center for Infectious Disease Research in Zambia; Lusaka, Zambia

${ }^{8}$ HIV Prevention Research Unit, South Africa Medical Research Council; Durban, South Africa

${ }^{9}$ Division of Infectious Diseases, University of North Carolina; Chapel Hill, NC, USA

${ }^{10}$ Centre for the AIDS Program of Research in South Africa, Doris Duke Medical Research Institute, Nelson R. Mandela School of Medicine, University of KwaZulu-Natal; Congella, South Africa

${ }^{11}$ Department of Epidemiology, Mailman School of Public Health, Columbia University; New York, NY, USA

\section{Abstract}

Background-The vaginal microbiota may play a role in mediating susceptibility to sexually transmitted infections, including Trichomonas vaginalis (TV).

Methods-Data were analyzed from HIV-1 seronegative women participating in HIV Prevention Trials Network Protocol 035. At quarterly visits for up to 30 months, participants completed

Correspondence: Jennifer E. Balkus, PhD, MPH, Fred Hutchinson Cancer Research Center, 1100 Fairview Ave N., M2-C200, P.O. Box 19024, Seattle, WA 98109-1024, Phone: +1 206667 7149, jbalkus@ fhcrc.org.

* Please address reprint requests to the corresponding author

This data was presented in part at the International Society for Sexually Transmitted Disease Research, held $14^{\text {th }}-17^{\text {th }}$ July 2013 in Vienna, Austria.

Potential Conflicts of Interest: All authors declare no commercial or other associations that might pose a conflict of interest relevant to the submitted work. 
structured interviews and specimens were collected for genital tract infection testing. TV was detected by saline microscopy. BV was characterized by Gram stain using the Nugent score $(\mathrm{BV}=7-10$; intermediate $=4-6$; normal $=0-3$ [reference group]). Cox proportional hazards models stratified by study site were used to assess the association between Nugent score category at the prior quarterly visit and TV acquisition.

Results-In this secondary analysis, 2,920 participants from Malawi, South Africa, USA, Zambia and Zimbabwe contributed 16,259 follow-up visits. BV was detected at 5,680 (35\%) visits and TV was detected at 400 (2.5\%) visits. Adjusting for age, marital status, hormonal contraceptive use, unprotected sex in the last week and TV at baseline, intermediate Nugent score and $\mathrm{BV}$ at the prior visit were associated with an increased risk of TV (intermediate score: adjusted hazard ratio $[\mathrm{aHR}]=1.73,95 \%$ confidence interval $[\mathrm{CI}] 1.21-2.19 ; \mathrm{BV}: \mathrm{aHR}=2.40,95 \%$ CI 1.92-3.00). Sensitivity analyses excluding 211 participants with TV at baseline were similar to those from the full study population (intermediate score: aHR=1.54, 95\% CI 1.10-2.14; BV: $\mathrm{aHR}=2.23,95 \%$ CI $1.75-2.84$ )

Conclusions-Women with a Nugent score $>3$ were at an increased risk of acquiring TV. If this relationship is causal, interventions that improve the vaginal microbiota could contribute to reductions in TV incidence.

\section{Keywords}

Bacterial vaginosis; Trichomonas vaginalis; vaginal microbiota; sexually transmitted disease acquisition; prospective cohort

\section{Introduction}

Bacterial vaginosis (BV) is a common vaginal syndrome that affects hundreds of millions of women globally each year [1,2]. The presence of BV has been associated with a number of adverse reproductive health outcomes [2], including an increased risk of sexually transmitted infections (STIs) [3-8]. Trichomonas vaginalis is one of the most common curable STIs [9]. It is frequently detected among women with BV [10-12] and is also associated adverse reproductive health outcomes including preterm birth, low birth weight, and HIV acquisition [13,14]. There is mounting evidence that the vaginal microbiota may play a role in mediating susceptibility to STIs, including TV. It is hypothesized that vaginal lactobacilli, the predominant bacterial species detected among women with a healthy vaginal environment, may utilize several mechanisms to protect against genital pathogen colonization [15]. Lactic acid production contributes to maintaining a lower vaginal $\mathrm{pH}$, which may inhibit pathogen colonization [16]. In addition, exposure to hydrogen peroxide $\left(\mathrm{H}_{2} \mathrm{O}_{2}\right)$ producing Lactobacillus species in vitro has been shown to decrease activity of BVassociated bacteria [17] and other genital tract pathogens [18]. Findings from in vitro studies are supported by data from epidemiologic studies, which showed that the absence or low levels of Lactobacillus colonization are associated with TV infection [19, 20].

Conversely, several prospective studies have reported an increased risk of TV among women with an abnormal vaginal microbiota or BV [3, 4, 6-8]. However, published studies that have assessed the association between the vaginal microbiota and TV acquisition have 
defined "normal" and "abnormal" vaginal microbiota in a variety of ways, making it challenging to compare results across studies. We sought to evaluate the association between the vaginal microbiota at the prior study visit and incident TV infection among women enrolled in a biomedical HIV prevention trial using several categorizations of the vaginal microbiota.

\section{Materials and Methods}

This is a secondary analysis of data from women enrolled in HIV Prevention Trials Network (HPTN) Protocol 035, a phase II/IIB, four-arm, multisite, randomized, controlled trial comparing BufferGel and 0.5\% PRO 2000 gel against two comparator arms (HEC placebo gel and no gel) for prevention of HIV infection (Clinicaltrials.gov \#NCT00074425). Detailed methods for the trial have been described previously [21]. Briefly, between February 2005 and October 2008, 3,087 HIV-1 uninfected women from five countries (Malawi, South Africa, United States of America [USA], Zambia, and Zimbabwe) were enrolled and followed for a minimum of 12 months and a maximum of 30 months, depending on the date of enrollment. Eligible women were $\geq 18$ years of age, HIV-1 seronegative, non-pregnant and sexually active (reported vaginal intercourse at least once in the past three months). Women were not eligible to participate if they reported a history of an adverse reaction to latex, history of non-therapeutic injection drug use in the past 12 months, frequent vaginal intercourse (more than two times per day in the prior two weeks), were within six weeks of the last pregnancy outcome, or had plans to become pregnant during follow-up. All institutional review boards and relevant regulatory authorities approved the trial at each site and all participants provided written informed consent.

Participants were randomly assigned in equal proportions to one of the four study arms. Study gels were dispensed in single-use, pre-filled applicators and were similar in appearance. Participants in the three gel arms were advised to use condoms and instructed to insert a single dose of gel intravaginally up to 1 hour prior to each act of vaginal intercourse, while participants in the no gel arm were advised to use condoms. At each monthly followup visit, a urine pregnancy test was performed. At quarterly visits, data were collected on self-reported gel use, condom use, sexual behaviors, vaginal washing and contraceptive use. Speculum-assisted pelvic examinations were performed with collection of specimens for diagnosis of genital tract infections and blood was collected through venipuncture for HIV-1 testing. All participants received a comprehensive HIV prevention package that included ongoing HIV risk reduction counseling, free male latex condoms, and diagnosis and treatment of STIs throughout the trial.

\section{Laboratory procedures}

A Gram stain of vaginal fluid was evaluated at a central laboratory for diagnosis of BV using the Nugent score [22]. A vaginal saline wet mount was examined microscopically for the presence of motile trichomonads for diagnosis of TV. Urine specimens were tested for Chlamydia trachomatis and Neisseria gonorrhoeae annually and at study exit using BD ProbeTec ET (Becton Dickinson; Franklin Lakes, New Jersey, USA). HIV-1 infection status was determined using a standardized algorithm. The Determine HIV 1/2 (Abbott Diagnostic 
Division; Hoofddorp, Netherlands) test was used with either the OraQuick® (Orasure Technologies; Bethlehem, Pennsylvania, USA), or Uni-Gold Recombigen ${ }^{\circledR}$ HIV test (Trinity Biotech; Wicklow, Ireland). Western blot (Genetics systems HIV-1 Western Blot kit, BioRad Laboratories; Hercules, CA, USA) was performed on samples with any positive HIV-1 result. HSV-2 testing was performed at enrollment and study exit using the HerpeSelect-2 EIA (Focus Technologies; Cypress, California, USA).

\section{Statistical analysis}

The objective of this analysis was to assess the association between the vaginal microbiome at the quarterly visit prior to TV testing and first incident TV infection. The vaginal microbiome was characterized using the Nugent score, where a score of 7-10 indicated BV, 4-6 was considered intermediate and 0-3 was considered normal (reference group). In our primary analysis, we separately compared incident TV infections among women with a Nugent score of 7-10 and 4-6 versus 0-3. Secondary analyses included comparisons of incident TV among women with a Nugent score $>3$ versus $0-3$ and a Nugent score $>6$ versus $0-6$, as these comparisons have been utilized by others [3, 4, 6-8]. Participants were excluded from the analysis if they did not return for a follow-up visit after enrollment, did not receive TV testing during follow-up or if they were found to be HIV-1 infected at baseline. If Nugent score results were not available at the quarterly visit prior to TV testing, the TV result visit was excluded from the analysis. Log-binomial regression with generalized estimating equations using an independent correlation structure and robust standard errors was used to assess demographic, behavioral and clinical factors associated with BV. Cox proportional hazards models stratified by study site were used to assess the association between the Nugent score at the prior quarterly visit and TV acquisition. Age, marital status, unprotected sex in the past week and baseline TV status were included in the multivariable model based on a priori assumptions. Additional factors were considered for inclusion in the model if they were associated with BV by Nugent score and incident TV infection $(\mathrm{p}<0.10)$. Participants were censored at their first incident TV infection or if they became pregnant, HIV-1 infected or tested positive for another STI (C. trachomatis or N. gonorrhoeae). Since participants received treatment for STIs diagnosed at enrollment, we included participants diagnosed with TV at baseline in the primary analysis. However, among women with TV at baseline, subsequent infections may represent TV persistence or re-infection; therefore, we conducted a sensitivity analysis excluding participants who tested positive for TV at enrollment. All statistical tests were assessed using a 2-sided a of 0.05. Analyses were conducted using Stata version 12.0 (StataCorp, Inc., College Station, TX).

\section{Results}

Of 3,087 HIV-1-uninfected participants enrolled in HPTN 035, 71 (2\%) participants did not return for follow-up, did not undergo TV testing during follow-up or did not have BV results in the interval prior to all TV testing during follow-up. In addition, 96 (3\%) participants were censored for other reasons prior to having a TV test with BV results during follow-up and were excluded ( 81 were censored due to pregnancy, 11 were HIV-infected, and 4 were diagnosed with another STI), leaving 2,920 (95\%) participants who contributed 16,259 follow-up visits for analysis. Compared to participants included in the analysis, participants 
who were excluded were slightly younger (median age [interquartile range (IQR)]: 23 years $(21-27)$ versus 25 years $(22-30) ; \mathrm{p}<0.001)$ and less likely to be married or living with a partner (58\% versus 70\%; $\mathrm{p}=0.007)$. Sexual behaviors reported at baseline, BV and STIs were similar between participants who were included versus those excluded from the analysis (data not shown).

Demographic, behavioral, and clinical characteristics at enrollment are presented in aggregate and by TV acquisition status during follow-up in Table 1 . The median number of follow-up visits per participant was 4 (IQR: 3-6). BV by Nugent score was detected at 5,680 $(35 \%)$ of 16,259 visits and an intermediate score was present at 2,622 (16\%) visits. Being married or living with a partner was associated with a decreased likelihood of BV during follow-up (odds ratio $[\mathrm{OR}]=0.90 ; 95 \%$ confidence interval $[\mathrm{CI}] 0.83,0.98$ ). In addition, compared to participants not using hormonal contraception, oral hormonal contraception and injectable hormonal contraception were both associated with modest decreases in the likelihood of BV during follow-up (oral contraceptive use: OR=0.90; 95\% CI 0.80, 0.99; injectable use: $\mathrm{OR}=0.77 ; 95 \% \mathrm{CI} 0.70,0.84)$. Conversely, $\mathrm{TV}$ at baseline was associated with an increased likelihood of BV (OR=1.19; 95\% CI 1.04, 1.36). Self-reported sexual behaviors (number of sex partners in the past 3 months, vaginal sex in the past week, unprotected sex in the past week), vaginal washing practices, and study arm were not associated with BV during follow-up (data not shown).

Overall, TV was detected at 400/16,259 (2.5\%) visits; TV was detected at 1.5\% $(118 / 7,957)$ of visits with a normal Nugent score at the prior visit compared to $2.6 \%(67 / 2,622)$ among visits with an intermediate score at the prior visit and 3.8\% (215/5,680) among women with $\mathrm{BV}$ at the prior visit. In univariate analyses, higher Nugent score category at the prior visit was associated with an increased risk TV infection (Table 2). An intermediate score was associated with a 1.73-fold increased likelihood of TV (95\% CI 1.28, 2.33), while BV was associated with a 2.53 -fold increased likelihood (95\% CI 2.02, 3.17). This linear trend in the hazard ratios was statistically significant $(\mathrm{p}<0.001)$. After adjusting for age, marital status, hormonal contraceptive use, unprotected sex in the last week and TV at baseline, an intermediate score and BV at the prior visit were independently associated with an increased likelihood of TV (Table 2). Secondary analyses using alternative categorizations of the Nugent score showed similar results (Nugent score $>3$ versus $0-3$ : aHR $=2.15,95 \%$ CI 1.74 , 2.67 ; Nugent score $>6$ versus $0-6$ : aHR $=2.06,95 \%$ CI 1.69, 2.51).

Baseline TV was also associated with an increased risk of TV in our multivariable model (aHR=2.65; 95\% CI 2.03, 3.48). Although participants diagnosed with TV at baseline received treatment according to the study protocol, subsequent infections may represent TV persistence due to treatment failure or re-infection if the partner was not treated (especially among participants who tested positive for TV at their first follow-up visit). Therefore, we conducted a sensitivity analysis excluding 211 participants who had baseline TV infection (39 [18\%] of which were also infected with TV at their next follow-up visit). Among 2,709 participants without TV infection at baseline, the likelihood of TV infection among women with abnormal vaginal microbiota was similar to results observed for the full study population for both intermediate Nugent score $(\mathrm{aHR}=1.54 ; 95 \% \mathrm{CI} 1.10,2.14)$ and BV $(\mathrm{aHR}=2.23 ; 95 \%$ CI $1.75,2.84)$. 


\section{Discussion}

Women with BV or an intermediate Nugent score were at an increased risk of acquiring TV compared to women with a normal vaginal microbiota. Results were similar when we assessed different categorizations of the Nugent score ( $>3$ versus $0-3$ and $>6$ versus $0-6$ ). The findings from our analyses extend those reported by others and add to the evidence that suggests that an abnormal vaginal microbiota increases susceptibility to TV infection $[3,4$, 6-8].

Although a number of studies have reported an increased risk of TV acquisition among women with $\mathrm{BV}$, the precise nature of the relationship between specific vaginal bacterial species and communities and TV susceptibility is not well understood. Over the past decade, molecular techniques targeting bacterial $16 \mathrm{~S}$ ribosomal RNA gene (rDNA) sequences have been used to characterize the vaginal microbiota [23], and hold great promise for providing insight into the relationships between vaginal health and STI acquisition, including TV. Improving our understanding of the relationship between the vaginal microbiota and TV susceptibility could lead to effective, targeted interventions that eradicate high-risk bacteria and promote vaginal colonization with bacterial species and communities that are associated with lower STI risk.

We also observed that women with TV infection at baseline had an increased likelihood of TV acquisition during follow-up, despite receiving treatment for TV at enrollment into the trial. The majority of women with TV at baseline tested negative for TV at their next followup visit. In addition, greater than $95 \%$ of women reported only 1 sexual partner during follow-up, suggesting that these women were re-infected with TV by their partners. Testing and treatment services were available to partners of women participating in the study; however, data on partner treatment was not systematically collected. Partner treatment services are not routinely offered in sub-Saharan Africa, where syndromic treatment is commonly used for STI treatment and microbiologic testing is not frequently performed [24]. As STI diagnostic capacity improves in resource-limited settings, additional information on the acceptability and uptake of partner treatment services in research studies and other clinical settings where pathogen specific STI testing and treatment occur as part of standard of care will be helpful for informing the scale-up of these services.

Compared to women not using hormonal contraception, we observed a decreased likelihood of TV among women using oral or injectable hormonal contraception. There is mixed evidence regarding the effect of hormonal contraception on TV acquisition. Several studies have reported a reduced risk of TV among women using oral or injectable hormonal contraception, while others have observed no association $[3,7,25,26]$. There are a number of methodological challenges that complicate analyses assessing the effect of hormonal contraception on STI acquisition, including choice of comparison group, method switching, and inadequate control of potential time-varying confounding factors such as condom use [27]. Differences in study design and analytic approach may contribute to the heterogeneity of published results. In the present analysis, the relationship between hormonal contraception and TV acquisition was assessed as a potential confounder and was not assessed as the primary objective of the study; therefore our findings should be interpreted 
in that context. Additional studies that appropriately address methodological challenges are needed to improve our understanding of the relationship between hormonal contraception and TV acquisition.

The present analysis includes several limitations that should be considered when interpreting the results. The primary limitation of this analysis is that only saline microscopy was used to detect TV infection. Although it is the most commonly used method for TV diagnosis in clinical settings, saline microscopy has considerably lower sensitivity compared with culture and nucleic acid amplification testing (NAAT) [28]. Use of a less sensitive diagnostic method for TV detection may have resulted in failure to detect TV infection in some women, which would contribute to an attenuation of the results. Furthermore, TV is more likely to be detected among women with a higher vaginal $\mathrm{pH}$ [13]. If organism burden differs by BV status, the low sensitivity of saline microscopy for TV detection may have resulted in greater TV detection among women with BV compared to those without. Thus, the relationship between BV and TV could be related to parasite density and its inherent impact on detection rather than differences in infection rates. Additional prospective studies using more sensitive diagnostics methods (NAAT) will help to further clarify the relationship between BV and TV acquisition. The interval between BV assessment and TV testing is also a limitation of this study. Evidence from prospective studies has shown that the vaginal microbiota fluctuates over time [29, 30]. Shorter intervals between assessment of $\mathrm{BV}$ and TV status, such as monthly testing, would reduce potential misclassification in the characterization of the vaginal microbiota prior to TV acquisition. In addition, our study population included women at risk for HIV infection participating in a clinical trial who may differ from women in the general population. Lastly, BV and STI acquisition are both associated with sexual activity [31]. Although the observed associations were strong and we attempted to control for confounding due to sexual behavior in our multivariable analyses, it is possible that a proportion of the observed association between BV and TV could be explained by insufficient control of important behavioral confounders.

In summary, women with an abnormal vaginal microbiota were at an increased risk of acquiring TV compared to women with a normal vaginal microbiota. We observed a doseresponse relationship, whereby the risk of TV acquisition increased with higher Nugent score category. If the relationship between the vaginal microbiota and TV acquisition is causal, interventions that decrease the incidence of BV and promote a normal vaginal microbiota could potentially contribute to reductions in TV incidence. However, current BV treatments fail to produce sustained changes in the vaginal microbiota [32-34]. Alternative regimens that improve cure rates and produce sustained changes in the vaginal microbiota are needed. Such interventions that successfully cure and control BV could be evaluated in clinical trials as part of a vaginal health approach to reducing susceptibility to TV infection.

\section{Acknowledgments}

We sincerely thank the women who participated in this study. We gratefully acknowledge the HPTN 035 study team and study sites for their work on data and sample collection and the Statistical Center for their work on data management.

Funding: HIV Prevention Trials Network (HPTN) Protocol 035 was funded by the US National Institutes of Health (NIH). The trial was designed and implemented by the HPTN and the Microbicide Trials Network (MTN). The 
HPTN (U01AI4674) has been funded by the National Institute of Allergy and Infectious Diseases (NIAID), the Eunice Kennedy Shriver National Institute of Child Health and Human Development (NICHD), the National Institute of Drug Abuse (NIDA), and the National Institute of Mental Health (NIMH). The MTN (UM1 AI068633) has been funded by NIAID, NICHD, and NIMH. The Statistical Center was supported by NIAID (UM1AI068615).

\section{References}

1. Allsworth JE, Peipert JF. Prevalence of bacterial vaginosis: 2001-2004 National Health and Nutrition Examination Survey data. Obstet Gynecol. 2007; 109:114-120. [PubMed: 17197596]

2. Hillier, S. Bacterial vaginosis. In: Holmes, KK.; S, P.; Stamm, W.; Piot, P.; Wasserheit, J.; Corey, L.; Cohen, M.; Watts, D., editors. Sexually Transmitted Diseases. New York: McGraw-Hill; 2008.

3. McClelland RS, Sangare L, Hassan WM, Lavreys L, Mandaliya K, Kiarie J, et al. Infection with Trichomonas vaginalis increases the risk of HIV-1 acquisition. J Infect Dis. 2007; 195:698-702. [PubMed: 17262712]

4. Brotman RM, Klebanoff MA, Nansel TR, Yu KF, Andrews WW, Zhang J, et al. Bacterial vaginosis assessed by Gram stain and diminished colonization resistance to incident gonococcal, chlamydial, and trichomonal genital infection. J Infect Dis. 2010

5. Atashili J, Poole C, Ndumbe PM, Adimora AA, Smith JS. Bacterial vaginosis and HIV acquisition: a meta-analysis of published studies. AIDS. 2008; 22:1493-1501. [PubMed: 18614873]

6. Martin HL, Richardson BA, Nyange PM, Lavreys L, Hillier SL, Chohan B, et al. Vaginal lactobacilli, microbial flora, and risk of human immunodeficiency virus type 1 and sexually transmitted disease acquisition. J Infect Dis. 1999; 180:1863-1868. [PubMed: 10558942]

7. Kapiga S, Kelly C, Weiss S, Daley T, Peterson L, Leburg C, et al. Risk factors for incidence of sexually transmitted infections among women in South Africa, Tanzania, and Zambia: results from HPTN 055 study. Sex Transm Dis. 2009; 36:199-206. [PubMed: 19265734]

8. Rathod SD, Krupp K, Klausner JD, Arun A, Reingold AL, Madhivanan P. Bacterial vaginosis and risk for Trichomonas vaginalis infection: a longitudinal analysis. Sex Transm Dis. 2011; 38:882886. [PubMed: 21844747]

9. WHO. Prevalence and incidence of selected sexually transmitted infections. Chlamydia trachomatis, Neisseria gonorrhoeae, syphilis and Trichomonas vaginalis: methods and results used by WHO to generate 2005 estimates. 2011

10. Gatski M, Martin DH, Clark RA, Harville E, Schmidt N, Kissinger P. Co-occurrence of Trichomonas vaginalis and bacterial vaginosis among HIV-positive women. Sex Transm Dis. 2011; 38:163-166. [PubMed: 20842073]

11. Madhivanan P, Bartman MT, Pasutti L, Krupp K, Arun A, Reingold AL, et al. Prevalence of Trichomonas vaginalis infection among young reproductive age women in India: implications for treatment and prevention. Sex Health. 2009; 6:339-344. [PubMed: 19917204]

12. Franklin TL, Monif GR. Trichomonas vaginalis and bacterial vaginosis. Coexistence in vaginal wet mount preparations from pregnant women. J Reprod Med. 2000; 45:131-134. [PubMed: 10710744]

13. Hobbs, M.; Sena, A.; Swygard, H.; Schwebke, J. Trichomonas vaginalis and Trichomoniasis. In: Holmes, KK.; S, P.; Stamm, W.; Piot, P.; Wasserheit, J.; Corey, L.; Cohen, M.; Watts, D., editors. Sexually Transmitted Diseases. New York: McGraw-Hill; 2008.

14. Schwebke JR, Burgess D. Trichomoniasis. Clin Microbiol Rev. 2004; 17:794-803. [PubMed: 15489349]

15. Spurbeck RR, Arvidson CG. Lactobacilli at the front line of defense against vaginally acquired infections. Future Microbiol. 2011; 6:567-582. [PubMed: 21585263]

16. Boskey ER, Telsch KM, Whaley KJ, Moench TR, Cone RA. Acid production by vaginal flora in vitro is consistent with the rate and extent of vaginal acidification. Infect Immun. 1999; 67:51705175. [PubMed: 10496892]

17. Atassi F, Brassart D, Grob P, Graf F, Servin AL. Lactobacillus strains isolated from the vaginal microbiota of healthy women inhibit Prevotella bivia and Gardnerella vaginalis in coculture and cell culture. FEMS Immunol Med Microbiol. 2006; 48:424-432. [PubMed: 17059467] 
18. Saigh JH, Sanders CC, Sanders WE Jr. Inhibition of Neisseria gonorrhoeae by aerobic and facultatively anaerobic components of the endocervical flora: evidence for a protective effect against infection. Infect Immun. 1978; 19:704-710. [PubMed: 415983]

19. Torok MR, Miller WC, Hobbs MM, Macdonald PD, Leone PA, Schwebke JR, et al. The association between Trichomonas vaginalis infection and level of vaginal lactobacilli, in nonpregnant women. J Infect Dis. 2007; 196:1102-1107. [PubMed: 17763335]

20. Baeten JM, Hassan WM, Chohan V, Richardson BA, Mandaliya K, Ndinya-Achola JO, et al. Prospective study of correlates of vaginal Lactobacillus colonisation among high-risk HIV-1 seronegative women. Sex Transm Infect. 2009; 85:348-353. [PubMed: 19329442]

21. Abdool Karim SS, Richardson BA, Ramjee G, Hoffman IF, Chirenje ZM, Taha T, et al. Safety and effectiveness of BufferGel and 0.5\% PRO2000 gel for the prevention of HIV infection in women. AIDS. 2011; 25:957-966. [PubMed: 21330907]

22. Nugent RP, Krohn MA, Hillier SL. Reliability of diagnosing bacterial vaginosis is improved by a standardized method of gram stain interpretation. J Clin Microbiol. 1991; 29:297-301. [PubMed: 1706728]

23. Fredricks DN, Fiedler TL, Marrazzo JM. Molecular identification of bacteria associated with bacterial vaginosis. N Engl J Med. 2005; 353:1899-1911. [PubMed: 16267321]

24. Golden MR, Estcourt CS. Barriers to the implementation of expedited partner therapy. Sex Transm Infect. 2011; 87(Suppl 2):ii37-38. [PubMed: 22110153]

25. Torok M, Miller W, Hobbs M, MacDonald P, Leone P, Schwebke J, et al. The association between oral contraceptives, depot-medroxyprogesterone acetate, and trichomoniasis. Sexually Transmitted Diseases June. 2009; 36:336-340.

26. Baeten JM, Nyange PM, Richardson BA, Lavreys L, Chohan B, Martin HL Jr, et al. Hormonal contraception and risk of sexually transmitted disease acquisition: results from a prospective study. Am J Obstet Gynecol. 2001; 185:380-385. [PubMed: 11518896]

27. Polis C, Westreich D, Balkus J, Heffron R. Assessing the effect of hormonal contraception on HIV acquisition in observational data: challenges and recommended analytic approaches. AIDS. 2013; (Suppl 1):S35-43. [PubMed: 24088682]

28. Nye MB, Schwebke JR, Body BA. Comparison of APTIMA Trichomonas vaginalis transcriptionmediated amplification to wet mount microscopy, culture, and polymerase chain reaction for diagnosis of trichomoniasis in men and women. Am J Obstet Gynecol. 2009; 200:188, e181-187. [PubMed: 19185101]

29. Fredricks DN. Molecular methods to describe the spectrum and dynamics of the vaginal microbiota. Anaerobe. 2011; 17:191-195. [PubMed: 21376827]

30. Srinivasan S, Liu C, Mitchell CM, Fiedler TL, Thomas KK, Agnew KJ, et al. Temporal variability of human vaginal bacteria and relationship with bacterial vaginosis. PLoS One. 2010; 5:e10197. [PubMed: 20419168]

31. Fethers KA, Fairley CK, Hocking JS, Gurrin LC, Bradshaw CS. Sexual risk factors and bacterial vaginosis: a systematic review and meta-analysis. Clin Infect Dis. 2008; 47:1426-1435. [PubMed: 18947329]

32. Sobel JD, Ferris D, Schwebke J, Nyirjesy P, Wiesenfeld HC, Peipert J, et al. Suppressive antibacterial therapy with $0.75 \%$ metronidazole vaginal gel to prevent recurrent bacterial vaginosis. Am J Obstet Gynecol. 2006; 194:1283-1289. [PubMed: 16647911]

33. Balkus JE, Jaoko W, Mandaliya K, Richardson BA, Masese L, Gitau R, et al. The posttrial effect of oral periodic presumptive treatment for vaginal infections on the incidence of bacterial vaginosis and Lactobacillus colonization. Sex Transm Dis. 2012; 39:361-365. [PubMed: 22504600]

34. Bradshaw CS, Morton AN, Hocking J, Garland SM, Morris MB, Moss LM, et al. High recurrence rates of bacterial vaginosis over the course of 12 months after oral metronidazole therapy and factors associated with recurrence. J Infect Dis. 2006; 193:1478-1486. [PubMed: 16652274] 
Table 1

Participant characteristics at enrollment into HPTN 035*

\begin{tabular}{|c|c|c|c|}
\hline & $\begin{array}{l}\text { All participants } \\
\qquad=\mathbf{N}, 920\end{array}$ & $\begin{array}{l}\text { Participants who acquired TV } \\
\text { infection during follow-up } \\
\qquad \mathrm{N}=400\end{array}$ & $\begin{array}{c}\text { Participants who did not acquire TV } \\
\text { infection during follow-up } \\
\mathrm{N}=\mathbf{2 , 5 2 0}\end{array}$ \\
\hline \multicolumn{4}{|l|}{ Demographic characteristics } \\
\hline \multicolumn{4}{|l|}{ Age } \\
\hline$<25$ years & $1,303(45)$ & $174(44)$ & $1,129(45)$ \\
\hline $25-29$ years & $840(29)$ & $94(23)$ & $746(30)$ \\
\hline $30-34$ years & $532(18)$ & $68(17)$ & $464(18)$ \\
\hline $35-39$ years & $112(4)$ & $32(8)$ & $80(3)$ \\
\hline$>40$ years & $133(5)$ & $32(8)$ & $101(4)$ \\
\hline Married or co-habiting & $2,041(70)$ & $247(62)$ & $1,794(71)$ \\
\hline Some secondary school & $1,832(63)$ & $207(52)$ & $1,625(64)$ \\
\hline \multicolumn{4}{|l|}{ Site } \\
\hline Malawi - Blantyre & 404 (14) & $42(11)$ & $362(14)$ \\
\hline Malawi - Lilongwe & $574(20)$ & $115(29)$ & $459(18)$ \\
\hline South Africa - Durban & $666(23)$ & $48(12)$ & $618(25)$ \\
\hline South Africa - Hlabisa & $317(11)$ & $96(24)$ & $221(9)$ \\
\hline USA - Philadelphia & $193(7)$ & $46(12)$ & $147(6)$ \\
\hline Zambia - Lusaka & $303(10)$ & $23(6)$ & $280(11)$ \\
\hline Zimbabwe - Chitungwiza & $248(8)$ & $16(4)$ & $232(9)$ \\
\hline Zimbabwe - Harare & $215(7)$ & $14(3)$ & $201(8)$ \\
\hline \multicolumn{4}{|l|}{ Study arm } \\
\hline No Gel & $728(25)$ & $97(25)$ & $629(25)$ \\
\hline Placebo & $728(25)$ & $99(25)$ & $631(25)$ \\
\hline PRO2000 & $733(25)$ & $117(27)$ & $616(24)$ \\
\hline BufferGel & $731(25)$ & $87(22)$ & $644(26)$ \\
\hline \multicolumn{4}{|l|}{ Behaviors } \\
\hline Vaginal sex in the past 7 days & $2,504(86)$ & $312(78)$ & $2,192(87)$ \\
\hline Condom use at last sex (\%) & $1,988(68)$ & $254(64)$ & $1,734(69)$ \\
\hline \multicolumn{4}{|l|}{ Vaginal washing $l$} \\
\hline Nothing & $1,800(62)$ & 275 (69) & $1,525(61)$ \\
\hline Water & $776(27)$ & $68(17)$ & $708(28)$ \\
\hline Soap \& water & $326(11)$ & $49(12)$ & $277(11)$ \\
\hline Vinegar \& water & $73(3)$ & $19(5)$ & $54(2)$ \\
\hline Paper, cotton, cloth, wool & $294(10)$ & $39(10)$ & $255(10)$ \\
\hline \multicolumn{4}{|l|}{ Hormonal contraception } \\
\hline Oral contraceptives & 567 (19) & $53(13)$ & $514(20)$ \\
\hline Injectables & $1,419(49)$ & $176(44)$ & $1,243(49)$ \\
\hline Implants & $37(1)$ & $5(1)$ & $32(1)$ \\
\hline \multicolumn{4}{|l|}{ Laboratory characteristics } \\
\hline Nugent score 7-10 (BV) & $1,082(37)$ & 197 (49) & $885(35)$ \\
\hline
\end{tabular}




\begin{tabular}{|c|c|c|c|}
\hline & $\begin{array}{l}\text { All participants } \\
\mathbf{N = 2 , 9 2 0}\end{array}$ & $\begin{array}{l}\text { Participants who acquired TV } \\
\text { infection during follow-up } \\
\qquad \mathrm{N}=400\end{array}$ & $\begin{array}{l}\text { Participants who did not acquire TV } \\
\text { infection during follow-up } \\
\qquad \mathrm{N}=\mathbf{2 , 5 2 0}\end{array}$ \\
\hline Nugent score 4-6 (intermediate) & $506(17)$ & $77(19)$ & $429(17)$ \\
\hline Trichomonas vaginalis & $211(7)$ & $71(18)$ & $140(6)$ \\
\hline Chlamydia trachomatis $^{2}$ & $100(3)$ & $17(4)$ & $83(3)$ \\
\hline Neisseria gonorrhoeae ${ }^{2}$ & $20(1)$ & $1(<1)$ & $19(1)$ \\
\hline HSV-2 seropositive & $1,265(43)$ & $217(54)$ & $1,048(42)$ \\
\hline \multicolumn{4}{|l|}{ * Data presented as $\mathrm{N}(\%)$} \\
\hline \multicolumn{4}{|c|}{$\begin{array}{l}{ }^{1} \text { At enrollment, participants were asked about vaginal washing practices in the past month. Total }>100 \% \text { as participants could choose more than } \\
\text { one option. }\end{array}$} \\
\hline
\end{tabular}




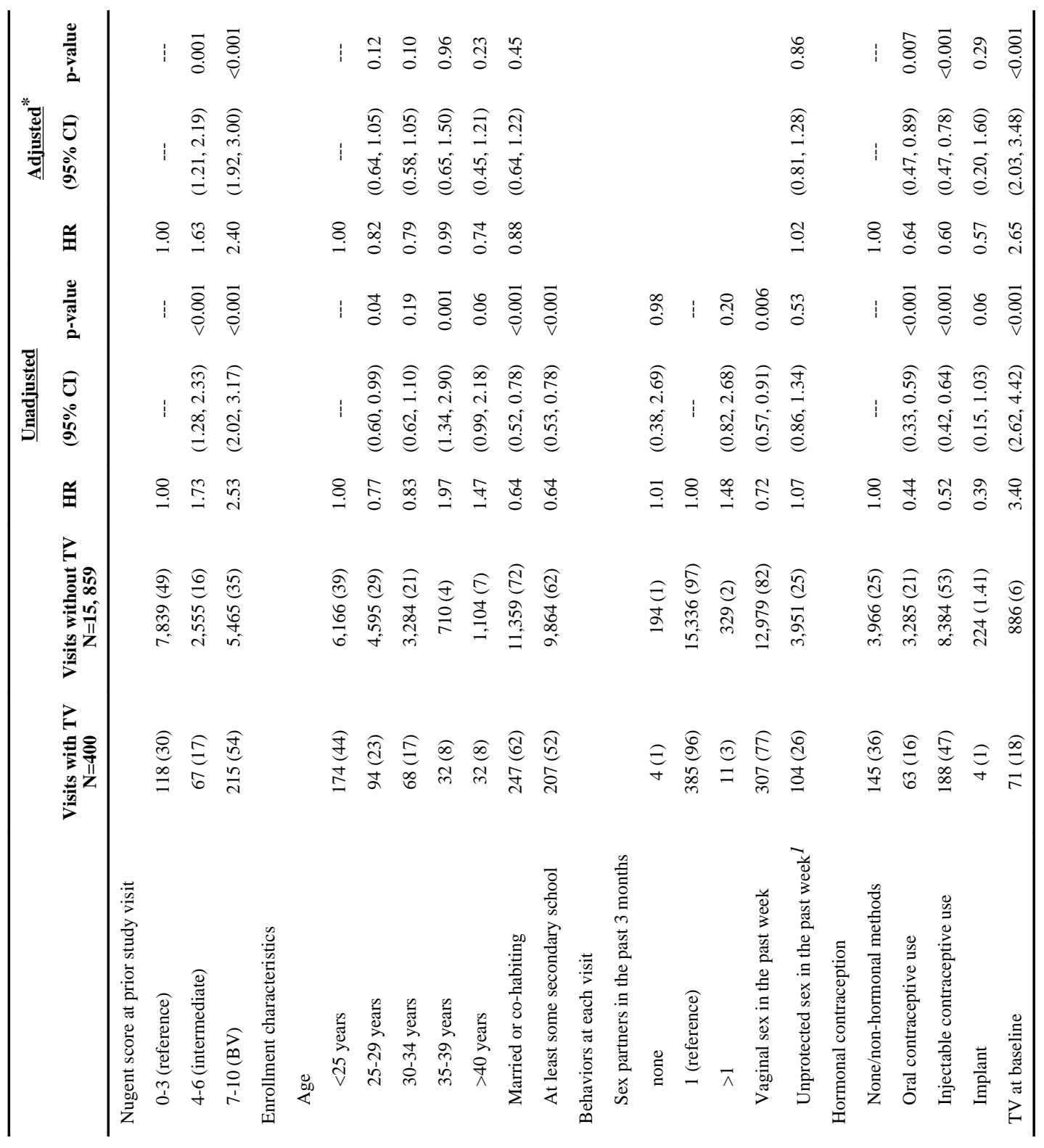


